\title{
Threat of drug resistant Staphylococcus aureus to health in Nepal
}

\author{
Shamshul Ansari, , Hari Prasad Nepal, Rajendra Gautam, Nabin Rayamajhi, Sony Shrestha, Goma Upadhyay, \\ Anju Acharya and Moti Lal Chapagain
}

\begin{abstract}
Background: Staphylococcus aureus is the most commonly isolated organism from the different clinical samples in hospital. The emergence and dissemination of methicillin resistant Staphylococcus aureus (MRSA) and growing resistance to non-beta-lactam antibiotics is making treatment of infections due to this organism increasingly difficult.

Methods: This study was conducted to determine the frequency of Staphylococcus aureus isolated from different clinical samples, rates of MRSA and full antibiotic susceptibility profiles. Clinical samples were cultured and Staphylococcus aureus was identified using standard microbiological methods recommended by the American Society for Microbiology (ASM). Methicillin resistance was confirmed using cefoxitin and oxacillin disks. Inducible clindamycin resistance was identified using D-zone test.

Results: From the processed samples, 306 isolates of Staphylococcus aureus were recovered. All the isolates were susceptible to vancomycin and teicoplanin. Methicillin resistance was observed in $43.1 \%$ of isolates while inducible clindamycin resistance in $12.4 \%$ of the isolates.

Conclusions: The results of our study reveals that rates of resistance to commonly prescribed antibiotics in Staphylococcus aureus clinical isolates is high. In particular, rate of methicillin resistance is alarming, prompting concern on the rational use of antibiotics and vigilant laboratory-based surveillance of resistance rates in Nepal.
\end{abstract}

Keywords: Staphylococcus aureus, MRSA, Inducible clindamycin resistance, Nepal

\section{Background}

Staphylococcus aureus is one of the most common human pathogens capable of causing a wide range of infections [1]. Over the past several decades, it has been a leading cause of both hospital and community-acquired infections [2]. It is associated with a variety of clinical infections including septicemia, pneumonia, wound sepsis, septic arthritis, osteomyelitis and post-surgical toxic shock syndrome with substantial rates of morbidity and mortality [3-6].

Increasing rates of antimicrobial resistance, often related to extensive use of antimicrobials, is resulting in fewer treatment options for bacterial infections. This problem is being identified across many different microorganisms, such as Pseudomonas spp. and Enterococcus

\footnotetext{
*Correspondence: shamshulansari483@yahoo.com

Department of Microbiology, Chitwan Medical College, Bharatpur, Chitwan,
} Nepal

\section{Biomed Central}

(c) 2014 Ansari et al.; licensee BioMed Central Ltd. This is an Open Access article distributed under the terms of the Creative Commons Attribution License (http://creativecommons.org/licenses/by/2.0), which permits unrestricted use, distribution, and reproduction in any medium, provided the original work is properly credited. spp [7]. Concerningly, rates of resistance to conventional antibiotics in Staphylococcus aureus has increased to high levels in some hospitals [8,9]. The incidence of community-acquired and hospital acquired Staphylococcus aureus infections has been rising with increasing emergence of drug-resistant strains called methicillin resistant Staphylococcus aureus (MRSA) [10-14]. MRSA now represents a global problem. Ever since its isolation, MRSA has emerged as one of the most common causes of hospital acquired infection and continues to remain as an important factor contributing to failure of management [15]. MRSA is frequently resistant to most of the commonly used antimicrobial agents including the aminoglycosides, macrolides, chloramphenicol, tetracycline and fluoroquinolones [16]. In addition, MRSA strains should be considered to be resistant to all cephalosporins, cephems and other beta-lactams (such as ampicillinsulbactam, amoxicillin-clavulanic acid, ticarcillin-clavulanic 
acid, piperacillin-tazobactam and the carbapenems) regardless of the in vitro test results obtained with those agents [17].

The objectives of this study, therefore, were to identify strains of Staphylococcus aureus from clinical samples and determine antimicrobial susceptibility profiles of these isolates.

\section{Methods}

A retrospective study was conducted from December 2010 to December 2012 at Chitwan Medical College Teaching Hospital (a 600 bed teaching hospital), Chitwan, Nepal. Patients were identified and data were extracted using the hospital information and support system.

\section{Sample collection}

The samples were collected in sterile containers by clinicians using aseptic technique and transported to the laboratory without delay. All samples were processed immediately.

\section{Culture and bacterial identification}

For the isolation and identification of Staphylococcus aureus, several media used were brain heart infusion (BHI) broth (for blood sample), blood agar (BA), chocolate agar (CHA), MacConkey agar (MA), DNase agar and mannitol salt agar (HiMedia Laboratories Pvt. Limited, India) and the tests used were catalase and coagulase. The collected samples were inoculated onto different culture media. The $\mathrm{CHA}$ plates were incubated in a $\mathrm{CO}_{2}$ incubator $\left(10 \% \mathrm{CO}_{2}\right)$ at $37^{\circ} \mathrm{C}$ for 24 hours. The BA and $\mathrm{MA}$ plates were incubated at $37^{\circ} \mathrm{C}$ for 24 hours in an aerobic atmosphere. Staphylococcus aureus was identified by standard microbiological techniques [18]. A purity plate was employed to ensure that the inoculum used for the biochemical tests was pure.

\section{Antibiotic susceptibility testing}

Antibiotic susceptibility tests of the Staphylococcus aureus isolates were performed by modified Kirby-Bauer disk diffusion method in compliance with Clinical and Laboratory Standards Institute (CLSI) guidelines using MuellerHinton agar standard media. The inhibition zone standards for antimicrobial susceptibility were considered from tables for interpretative zone diameters of CLSI [19]. Antibiotic disks (HiMedia Laboratories, Pvt. Limited, India) used were: penicillin $\mathrm{G}(10 \mathrm{U})$, ciprofloxacin $(5 \mu \mathrm{g})$, erythromycin $(15 \mu \mathrm{g})$, co-trimoxazole $(25 \mu \mathrm{g})$, gentamicin $(10 \mu \mathrm{g})$, amikacin $(30 \mu \mathrm{g})$, cephalexin $(30 \mu \mathrm{g})$, ceftriaxone $30 \mu \mathrm{g})$, cefoxitin $(30 \mu \mathrm{g})$, oxacillin $(1 \mu \mathrm{g})$, vancomycin $(30 \mu \mathrm{g})$, clindamycin $(2 \mu \mathrm{g})$ and teicoplanin $(30 \mu \mathrm{g})$. For the identification of MRSA strains, cefoxitin and oxacillin disks were used.

\section{Identification of methicillin resistant Staphylococcus aureus (MRSA) strains}

Methicillin resistant Staphylococcus aureus (MRSA) was identified by using oxacillin $(1 \mu \mathrm{g})$ and cefoxitin $(30 \mu \mathrm{g})$ disks. Plates were incubated at $35^{\circ} \mathrm{C}$. Plates containing oxacillin disk were read following a 24 hour incubation period. The diameter of the zone of inhibition (ZOI) of growth was recorded and interpreted as susceptible or resistant according to the criteria of CLSI. Staphylococcus aureus isolates were deemed methicillin resistant when the ZOI was $\leq 10 \mathrm{~mm}$ with the oxacillin disk or $\leq 21 \mathrm{~mm}$ with the cefoxitin disk [20].

\section{Identification of inducible clindamycin resistant strains} Inducible macrolide-lincosamide-streptogramin B (iMLSB) resistance was detected in Staphylococcus aureus by Disk approximation test placing a $2 \mu \mathrm{g}$ clindamycin disk $15 \mathrm{~mm}$ away from the edge of a $15 \mu \mathrm{g}$ erythromycin disk on a MHA plate. Following incubation, organisms that showed flattening of the clindamycin zone adjacent to the erythromycin disk (referred to as a "D" zone) were considered to exhibit inducible clindamycin resistance [20].

Staphylococcus aureus ATCC 25923 was used as a control organism for antibiotic sensitivity testing. For MRSA detection, Staphylococcus aureus ATCC 25923 and ATCC 43300 were used as negative and positive controls respectively.

\section{Ethical aspects}

The samples used in this study were from routine clinical specimens. Because acquiring the samples did not involve direct patient contact and did not interrupt routine clinical care, consent was not required. Permission to conduct the study was obtained from the Head of the Microbiology Department.

\section{Results \\ Isolate characteristics}

A total of 15718 samples (blood 7200, urine 7170, sputum 860 and pus 488) were processed from both inpatients and out-patients. Of 306 isolates of Staphylococcus aureus (175 from female patients and 131 from male patients), 120 (39.2\%), 83 (27.1\%), 63 (20.6\%) and 40 (13.1\%) were obtained from blood, pus, urine and sputum respectively. Among the processed samples the highest positivity rate was found in pus sample (17.0\%) followed by sputum (4.7\%), blood (1.7\%) and urine $(0.9 \%)$. The age distribution of the isolates is shown in Table 1.

\section{Antimicrobial susceptibility testing}

The antibiogram of the Staphylococcus aureus isolates is shown in Table 2. Interestingly, only vancomycin and teicoplanin retained $100 \%$ susceptibility. Almost all isolates 
Table 1 Distribution of clinical isolates of Staphylococcus aureus according to the age of patients $(n=306)$

\begin{tabular}{lc}
\hline Age of patients in years & Number of isolates (\%) \\
\hline $0-10$ & $98(32.0)$ \\
$11-20$ & $58(18.9)$ \\
$21-30$ & $72(23.3)$ \\
$31-40$ & $25(8.3)$ \\
$41-50$ & $10(3.4)$ \\
$51-60$ & $19(6.3)$ \\
$61-70$ & $19(6.3)$ \\
$71-80$ & $5(1.5)$ \\
Total & $\mathbf{3 0 6}(\mathbf{1 0 0})$ \\
\hline
\end{tabular}

(94.7\%) were resistant to penicillin, and most of them were resistant to other commonly used antibiotics. $43.1 \%$ of the isolates were resistant to methicillin by cefoxitin method, and $39.2 \%$ were resistant to oxacillin. Of the 100 isolates exhibiting erythromycin resistance, 38 (12.4\%) were found to have inducible clindamycin resistance by $\mathrm{D}$-zone test.

\section{Discussion}

Staphylococcus aureus gain access to the epidermis through cracks in the skin, abrasions, cuts, burns, surgical incisions and intravenous catheters causing wide spectrum of infections, from localized skin lesions such as abscesses, folliculitis to deep seated infections. In the present study, of 306 isolates of Staphylococcus aureus, 27\% were from pus sample, which signifies their important role in abscess formation.

Antimicrobial resistance has been noticed as one of the paramount microbial threats of the twenty-first century [21]. The multidrug resistance to most of the antibiotics used in infections caused by staphylococci is an increasing problem. The emergence of methicillin resistance among Staphylococcus aureus strains led to difficulties in the

Table 2 Antibiogram of Staphylococcus aureus $(n=306)$

\begin{tabular}{lc}
\hline Antimicrobial agents & Resistant isolates (\%) \\
\hline Penicillin & $290(94.7)$ \\
Cotrimoxazole & $250(81.7)$ \\
Cephalexin & $208(68.0)$ \\
Gentamicin & $185(60.4)$ \\
Ciprofloxacin & $195(63.7)$ \\
Erythromycin & $100(32.7)$ \\
Cefoxitin & $132(43.1)$ \\
Oxacillin & $120(39.2)$ \\
Clindamycin & $84(27.5)$ \\
Amikacin & $33(10.7)$ \\
Vancomycin & 0 \\
Teicoplanin & 0 \\
\hline
\end{tabular}

treatment of infections caused by this organism [22]. Therefore, surveillance on the antimicrobial susceptibility patterns of Staphylococcus aureus is of utmost importance in understanding new and emerging resistance trends as well as in the management of both hospital and community-acquired infections.

This study demonstrated that overall rates of susceptibility to commonly prescribed antibiotics in Staphylococcus aureus isolates were below 70\%, with the exception of clindamycin, amikacin, teicoplanin, and vancomycin. Despite the considerable progress in antimicrobial therapy, resistance in Gram-positive pathogens continues to increase, mainly in relation to the drugs commonly used in medical practice. A high proportion of isolates (94.7\%) were resistant to penicillin in this study. This was expected as it has been recognized that only a small proportion of the Staphylococcus aurues lineages do not produce beta-lactamases [23-26].

Erythromycin has been used extensively for the treatment of both minor and more serious staphylococcal infections. As a consequence, its role today is increasingly limited due to increasing resistance, which poses a great therapeutic challenge. One third (32.7\%) of our isolates were resistant to erythromycin, compared to previous similar studies in Nepal which have round resistance rates of $7.1 \%$ in $2010,11 \%$ in $2011,63.6 \%$ in 2013 [24,27,25]. Similarly, trimethoprim-sulfamethoxazole (co-trimoxazole) can be an alternative treatment choice, particularly for non-multi-resistant MRSA infections, although emergence of resistance has been previously observed. This may be due to excessive use of this drug for many other infections and over-the-counter availability of antimicrobials in the developing world for the treatment of many other infections. $81.7 \%$ of our isolates were resistant to co-trimoxazole, compared with $42.96 \%$ in 2009 [28], $12.5 \%$ in 2010 [26], 64\% in 2011 [27], and $72.7 \%$ in 2013 [25] in Nepal.

While ciprofloxacin is predominantly a Gram-negative drug, it does have activity against Staphylococcus aureus. As a consequence of low cost and easy availability, there has been indiscriminate use of ciprofloxacin in Nepal. We identified resistance rate of $63.7 \%$, much higher than previous studies (26\% in 2009 [23], 12\% in 2011 [27]). The same trend is seen with gentamicin, with $60.4 \%$ of our isolates being resistant compared with $46.98 \%$ in 2009 [28], 32.73\% in 2010 [26], $11 \%$ in 2011 [27], and $54.5 \%$ in 2013 [25]. Fortunately, rate of resistance to amikacin remains low.

All of our isolates had retained susceptibility to vancomycin and teicoplanin, consistent with previous studies $[23,25-27,29]$, confirming that glycopeptides should be used as empiric therapy for serious staphylococcal infections while waiting for susceptibility testing results to come through [30]. This is likely related to low usage of 
these agents in Nepal due to high cost. Concerningly, however, lineages with increased resistance to teicoplanin have been observed overseas [31-34].

Clindamycin is one of the drugs of choice in MRSA infections. Macrolide-resistant isolates of Staphylococcus aureus may have constitutive or inducible resistance to clindamycin (due to methylation of the 23S rRNA encoded by the erm gene also referred to as MLSB i.e., Macrolide, Lincosamide and type B Streptogramin resistance) or may be resistant only to macrolides (due to efflux mechanism encoded by the msrA gene) [20]. As the presence of an erm gene encoding for inducible resistance may result in treatment failure [35], it is important to perform its testing. We found 38 (12.4\%) D-test positive isolates, indicating inducible resistance to clindamycin.

Today the concern of MRSA has reached the pinnacle. It is noteworthy that MRSA can cause both community and hospital acquired infections. Prior antibiotic use is the most common risk factor for colonization and infection with MRSA. In our study, 43.1\% of Staphylococcus aureus isolates were found to be MRSA which is higher as compared to other studies conducted in Nepal. The incidence of MRSA was reported to be $20 \%$ in 2001 [36], $15.4 \%$ in 2005 [37], $26.14 \%$ in 2008 [29], 39.6\% in 2010 [26] and $42.4 \%$ in 2013 [25] in Nepal.

Two different methods were employed for the detection of MRSA. The cefoxitin disk method detected 132 (43.1\%) MRSA cases while the oxacillin disk method missed 12 cases and detected only 120 (39.2\%) Staphylococcus aureus as MRSA. According to CLSI [20], the cefoxitin disk test is comparable to the oxacillin disk test for the prediction of mecA-mediated resistance to oxacillin. The cefoxitin disk test is easier to read and thus is the preferred method. Besides, cefoxitin is an inducer of the mecA gene.

There are a number of factors contributing to increasing rates of resistance in Staphylococcus aureus in Nepal. Firstly, regulation of antibiotics is poor with their easy and over the counter availability without prescription. Some health care workers and pharmacists are often paid incentives by the pharmaceutical companies to prescribe or sell unnecessary antibiotics. Medical practice by unqualified personnel, who often prescribe unnecessary antibiotics, is yet other common problem in Nepal. Locally produced antibiotics are of questionable quality; and compliance of the patients is also often poor. Many antibiotics are prescribed without culture and sensitivity due to lack of laboratory facilities in most of the areas. Moreover, infection control policies are yet to be instituted properly in most of the hospitals and medical institutions of Nepal.

Thus, regular surveillance of hospital associated infections and antibiotic sensitivity pattern of MRSA; and formulation of definite antibiotic policy may be helpful for reducing the incidence of MRSA infection. Furthermore, healthcare workers should be trained to control hospital infection and infection control program should be conducted effectively in all health care centers.

\section{Conclusion}

This report demonstrates high rates of MRSA and resistance to other drugs in Staphylococcus aureus in our hospital. There is a need for longitudinal surveillance of MRSA and its antimicrobial susceptibility profile in Nepal. We recommend effective implementation of hospital infection control and antibiotic policies to control antibiotic resistance in Staphylococcus aureus.

\section{Competing interests}

The authors declare that they have no conflict of interest.

\section{Authors' contributions}

SA, HPN, RG, NR, AA and MLC conceived the design of the study. SA prepared the manuscript with help from HPN, SS and GU. RG, NR, AA and MLC supervised the work and manuscript. All authors read and approved the final manuscript.

\section{Acknowledgement}

We thank all the laboratory staffs of the Bacteriology Department of Chitwan Medical College Teaching Hospital (CMCTH) for their kind support in the collection of data and performing the necessary laboratory tests during the study.

Received: 7 May 2013 Accepted: 19 March 2014 Published: 22 March 2014

\section{References}

1. Foster TJ: The Staphylococcus aureus "superbug". J Clin Invest 2004, 114:1693-1696.

2. Lowy FD: Staphylococcus aureus infection. N England J Med 1998, 339:520-532.

3. Boyce JM: Epidemiology and prevention of nosocomial infections. In The staphylococci in human disease. Edited by Crossley KB, Archer GL. New York: Churchill Livingstone; 1997:309-329.

4. Shopsin B, Kreiswirth BN: Molecular epidemiology of methicillin-resistant Staphylococcus aureus. Emerg Infect Dis 2001, 7:323-326.

5. Cosgrove SE, Sakoulas G, Perencevich EN, Schwaber MJ, Karchmer AW, Carmeli Y: Comparison of mortality associated with methicillin resistant and methicillin sensitive Staphylococcus aureus bacteriemia: a meta analysis. Clin Infect Dis 2003, 36:53-59.

6. Engemann JJ, Carmeli Y, Cosgrove SE, Fowler VG, Bronstein MZ, Trivette SL, Briggs JP, Sexton DJ, Kaye KS: Adverse clinical and economic outcomes attributable to methicillin resistance among patients with Staphylococcus aureus surgical site infection. Clin Infect Dis 2003, 36:592-598.

7. Livermore DM: Introduction: the challenge of multiresistance. Int $\mathrm{J}$ Antimicrob 2007, 29:51-57.

8. Park DW, Kim MJ, Yang JA, Jeong HW, Sohn JW, Chun BC: Risk factors for isolation of low-level mupirocin-resistant versus susceptible methicillinresistant Staphylococcus aureus from patients in intensive care units. J Infect 2007, 54:337-342.

9. Manzur A, Vidal M, Pujol M, Cisnal M, Hornero A, Masuet C, Peña C, Gudiol F, Aziza J: Predictive factors of methicillin resistance among patients with Staphylococcus aureus bloodstream infections at hospital admission. J Hosp Infect 2007, 66:135-141

10. Steinberg JP, Clark CC, Hackman BO: Nosocomial and community acquired Staphylococcus aureus bacteremias from 1980 to 1993: impact of intravascular devices and methicillin resistance. Clin Infect Dis 1996, 23:255-259.

11. Emori TG, Gaynes RP: An overview of nosocomial infections, including the role of the microbiology laboratory. Clin Microbiol Rev 1993, 6:428-442.

12. Deresinski S: Methicillin-resistant Staphylococcus aureus: an evolutionary, epidemiologic, and therapeutic odyssey. Clin Infect Dis 2005, 40:562-573. 
13. Fluit AC, Wielders CL, Verhoef J, Schmitz FJ: Epidemiology and susceptibility of 3,051 Staphylococcus aureus isolates from 25 university hospitals participating in the European SENTRY study. J Clin Microbiol 2001, 39:3727-3732.

14. Herold BC, Immergluck LC, Maranan MC, Lauderdale DS, Gaskin RE, Boyle-Vavra S, Leitch CD, Daum RS: Community acquired methicillin-resistant Staphylococcus aureus in children with no identified predisposing risk. J American Med Assoc 1998, 279:593-598.

15. Salmenlinna S, Lyytikainen O, Vuopio-Varkila J: Community acquired methicillin-resistant Staphylococcus aureus, Finland. Emerging Infect Dis 2002, 8:602-607

16. Mandell G, Douglas J, Bennett R: Principles and practice of infectious disease 4th edition. Edinburgh, United Kingdome: Churchill Livingstone Ltd.; 1995.

17. National Committee for Clinical Laboratory Standards (NCCLS): Performance standards for antimicrobial susceptibility testing. 7th edition. Wayne PA, USA NCCLS; 2000. Vol. 20

18. HD I: Clinical microbiology procedures handbook. 2nd edition. ASM press: Washington DC; 2004.

19. Clinical and Laboratory Standard Institute (CLSI): Performance standards for antimicrobial susceptibility testing. Wayne, PA: USA:CLSI: M100-S16; 2006.

20. Clinical and Laboratory Standards Institute (CLSI): Performance standards for antimicrobial susceptibility testing, 17th informational supplement. Wayne, PA: USA:CLSI: M100-S17; 2007.

21. Smolinski MS, Hamburg MA, Lederberg J: Microbial threats to health: emergence, detection and response. Washington: Institute of Medicine; 2003. 32.

22. Waldvogel FA: Staphylococcus aureus (including staphylococcal toxic shock). In Mandell, Douglas and Bennett's principles and practice of infectious diseases. 5th edition. Edited by Mandell GL, Bennett JE, Dolin R. Philadelphia, PA: CV, A Harcourt Health Sciences Co; 2000.

23. Tiwari HK, Das AK, Sapkota D, Sivarajan K, Pahwa VK: Methicillin resistant Staphylococcus aureus: prevalence and antibiogram in a tertiary care hospital in western Nepal. J Infect Dev Ctries 2009, 3(9):681-684.

24. Shakya B, Shrestha S, Mitra T: Nasal carriage rate of methicillin resistant Staphylococcus aureus among at national medical college teaching hospital, Birgunj, Nepal. Nepal Med Coll J 2010, 12(1):26-29.

25. Mishra SK, Rijal BP, Pokhrel BM: Emerging threat of multidrug resistant bugs-Acinetobacter calcoaceticus baumannii complex and methicillin resistant Staphylococcus aureus. BMC Research Notes 2013, 6:98-103.

26. Sanjana RK, Shah R, Chaudhary N, Singh Yl: Prevalence and antimicrobial susceptibility pattern of methicillin-resistant Staphylococcus aureus (MRSA) in CMS-teaching hospital: a preliminary report. Journal of College of Medical Sciences-Nepal 2010, 6(1):1-6.

27. Baral $R$, Khanal $B$, Acharya A: Antimicrobial susceptibility patterns of clinical isolates of Staphylococcus aureus in Eastern Nepal. Health Renaissance 2011, 9(2):78-82.

28. Shrestha B, Pokhrel BM, Mohapatra TM: Antibiotic susceptibility pattern of nosocomial isolates of Staphylococcus aureus in a tertiary care hospital, Nepal. J Nepal Med Assoc 2009, 48(175):234-238.

29. Kumari N, Mohapatra TM, Singh Yl: Prevalence of methicillin-resistant Staphylococcus aureus (MRSA) in a tertiary-care hospital in Eastern Nepal. J Nepal Med Assoc 2008, 47(170):53-56.

30. Sader HS, Gales AC, Jones RN: Antimicrobial activity of linezolid against gram-positive cocci isolated in Brazil. Braz J Infect Dis 2001, 5:171-176.

31. Cormican $M G$, Jones RN: Emerging resistance to antimicrobial agents in gram-positive bacteria. Drugs 1996, 51:6-12.

32. Shittu AO, Lin J: Antimicrobial susceptibility patterns and characterization of clinical isolates of Staphylococcus aureus in Kwazulu-Natal province, South Africa. BMC Infect Dis 2006, 6:125-137.

33. Centers for Disease Control and Prevention: Staphylococcus aureus resistance to vancomycin in the United States of America. MMWR 2002, 51:565-567.

34. Classen M, Nouwe J, Fang N, Ott A, Verbrugh H, Hofman A, Vanbelkum A, Uitterlinder A: Staphylococcus aureus nasal carriage is not associated with known polymorphism in the vitamin D receptor gene. FEMS Immunol Med Microbiol 2005, 43:173-176.
35. Lim HS, Lee $H$, Roh KH, Yum JH, Yong D, Lee K, Chong Y: Prevalence of inducible clindamycin resistance in Staphylococcal isolates at a Korean tertiary care hospital. Yonsei Med J 2006, 47:480-484.

36. Rai CKS: Antibiotic susceptibility patters of Staphylococcus aures. J Nepal MedicalAssociation 2001, 40:138.

37. Subedi S, Brahmadarhan KN: Antimicrobial susceptibility pattern of clinical isolates of Staphylococcus aureus in Nepal. Clinical microbiology infect 2005, 11(3):253-237.

doi:10.1186/1471-2334-14-157

Cite this article as: Ansari et al:: Threat of drug resistant Staphylococcus aureus to health in Nepal. BMC Infectious Diseases 2014 14:157.

\section{Submit your next manuscript to BioMed Central and take full advantage of:}

- Convenient online submission

- Thorough peer review

- No space constraints or color figure charges

- Immediate publication on acceptance

- Inclusion in PubMed, CAS, Scopus and Google Scholar

- Research which is freely available for redistribution

Submit your manuscript at www.biomedcentral.com/submit
C Biomed Central 